\title{
ZELEVINSKI ALGEBRAS RELATED TO PROJECTIVE REPRESENTATIONS
}

\author{
M. BEAN AND P. HOFFMAN
}

\begin{abstract}
We define $L$-PSH-algebras, and prove a classification theorem for such objects. The letters refer respectively to a ground ring $L$ and to the positivity, selfadjointness and Hopf structures on an algebra, the basic example of which occurred in the study of projective representations of $S_{n}$. This is analogous to an idea over $\mathbf{Z}$ due to Zelevinski in connection with linear representations.
\end{abstract}

In $[\mathbf{Z}$, Chapter I], Zelevinski introduced PSH-algebras and applied them to the ordinary representation theory of the symmetric group, monomial groups and matrix groups. See also $[\mathbf{H}$, pp. 81-86] and [L] for related less comprehensive results. A PSH-algebra is a commutative $\mathbf{Z}$-algebra with several pieces of extra structure:

(i) a grading over the nonnegative integers $\mathbf{N}$;

(ii) a comultiplication making it into a Hopf algebra;

(iii) a specified basis as a free $\mathbf{Z}$-module. Appropriate axioms relate these structures.

Zelevinski gave a complete classification of PSH-algebras, each being isomorphic to a tensor product of atoms. There are countably many atoms, differing from each other only by "simple regrading" - that is, multiplying all gradings by a fixed positive rational. As (ungraded) rings all atoms are $\mathbf{Z}\left[x_{1}, x_{2}, \ldots\right]$, with $\operatorname{deg} x_{i}=$ $i \operatorname{deg} x_{1}$. Representation theorists interpret this object as $\oplus R\left(S_{i}\right)$ (with $R\left(S_{i}\right)$ in the $i$ th grading if $\operatorname{deg} x_{1}=1$ ); algebraic topologists as $H^{*}(B U)$ (with degree as dimension, the Chern class $x_{i}$ having degree $2 i$ ); and undergraduates as the ring of stable symmetric functions (with degree as the usual polynomial degree).

In [H-H], John Humphreys and the second author introduced an algebra structure in the graded group whose components are the Grothendieck groups of "negative representations" of certain double covers $\tilde{S}_{n}$ and $\tilde{A}_{n}$ of the symmetric and alternating groups. Except for a few small $n$, these groups have elements which can be interpreted as formal differences of the projective representations of $S_{n}$ and $A_{n}$. More generally for finite $G$, the coverings $\widetilde{G ? S_{n}}$ and $\widetilde{G ? A_{n}}$ yield an object which is a tensor product of atoms, each atom isomorphic to a simple regrading of the above object (which is the object obtained when $G$ is trivial).

This suggests that there may be an abstract notion and structure theorem analogous to Zelevinski's (and perhaps a common generalization but that is not pursued here). Below we give the appropriate definition, and prove a structure theorem. Essentially what must be done is to change the ground $\operatorname{ring} \mathbf{Z}$ to a certain $\mathbf{Z} / 2$-graded

Received by the editors March 12, 1987.

1980 Mathematics Subject Classification (1985 Revision). Primary 16A24; Secondary 20C25, $20 \mathrm{C} 30$.

Partially supported by NSERC grant A4810. 
ring $L$, and to chanre the $\mathbf{N}$-grading to a $(\mathbf{Z} / 2 \times \mathbf{N})$-grading of abelian groups (in fact it is an $\mathbf{N}$-grading of $\mathbf{Z} / 2$-graded $L$-modules).

In $\S 1$ we give a slight generalization of Zelevinski's (strictly commutative) result to the graded commutative situation. Here, up to simple regrading, there are two atoms

(i) $\mathbf{Z}\left[x_{1}, x_{2}, x_{3}, \ldots\right]$; $\operatorname{dim} x_{i}=2 i n$, some $n>0$ (polynomial algebras as before, but degrees are even),

(ii) $\mathrm{Z}[y] / y^{2} ; \operatorname{dim} y=2 n-1$, some $n>0$ (exterior algebras on a single odd dimensional generator).

This helps to motivate our classification of atoms, where up to simple re-grading, there are four atoms, the structure depending on the biparity in $\mathbf{Z} / 2 \times \mathbf{Z} / 2$ of the bidimension in $\mathbf{Z} / 2 \times \mathbf{N}$ of the "bottom" generator.

In $\S 2$ we make the appropriate definitions, give a few general results, and state the theorems. In $\S 3$ we prove the decomposition theorem, and in $\S 4$, the structure theorem for atoms.

1. Zelevinski's theorem in the graded commutative category. Define a Z-PSH-algebra exactly as in [Z, pp. 12-18], except that the Hopf axiom:

$$
\Delta: R \rightarrow R \otimes R \text { is a ring homomorphism, }
$$

is qualified by requiring the multiplication in $R \otimes R$ to be $(x \otimes y) \cdot\left(x^{\prime} \otimes y^{\prime}\right)=$ $(-1)^{\operatorname{deg} x^{\prime} \operatorname{deg} y} x x^{\prime} \otimes y y^{\prime}$ (Zelevinski does not have the sign in his conventions). The analogue of Zelevinski's Proposition 1.6 (that associativity follows from the other axioms, as does commutativity) holds here with the same proof, except that commutativity is interpreted in the graded sense: $x y=(-1)^{\operatorname{deg}(x) \operatorname{deg}(y)} y x$. Furthermore his decomposition theorem [Z, p. 22] holds with no changes. Thus each Z-PSHalgebra is a graded tensor product of "atoms", where an atom by definition is a Z-PSH-algebra with a unique irreducible primitive element. In order to satisfy the modified $(\mathrm{H})$ above, the "atom" studied in $[\mathrm{Z}, \mathrm{pp} .27-42]$ must have all its nonzero elements in even degree. The analogous structure theorem for atoms holds modulo stating:

(i) In (g) [Z, p. 28] $R$ is unique up to isomorphism in being an atom whose irreducible primitive is in degree 2, and any atom whose irreducible primitive is in even degree is isomorphic to a simple regrading of $R$.

(ii) For any odd integer $2 n+1$ there is a unique atom with irreducible primitive of grading $2 n+1$. Its ring structure is $S=\mathbf{Z}[y] /\left(y^{2}=0\right)$, with comultiplication

$$
\Delta y=1 \otimes y+y \otimes 1,
$$

and with $\{1, y\}$ being the specified basis.

It is easily verified that $S$ is a a Z-PSH-algebra. It remains only to prove that $S$ is unique up to isomorphism, once $\operatorname{deg} y$ is fixed.

Let $S^{\prime}$ be an atom whose irreducible primitive $y^{\prime}$ has odd degree. Then $2 y^{\prime 2}=0$ by commutativity, so $y^{\prime 2}=0$ by freeness. All nonzero irreducibles $s^{\prime} \in S^{\prime}$ satisfy $\left\langle s^{\prime}, y^{\prime t}\right\rangle \neq 0$ for some $t$. Thus $S_{t}^{\prime}=0$ for all $t \neq 0$ or $\operatorname{deg} y^{\prime}$. By connectedness, $S_{0}^{\prime} \cong \mathbf{Z}$ generated by 1 . If $\operatorname{deg} s^{\prime}=\operatorname{deg} y^{\prime}$, then $\left\langle s^{\prime}, y^{\prime}\right\rangle \neq 0$. Thus $S_{\operatorname{deg} y^{\prime}}^{\prime}=\mathbf{Z}$ generated by $y^{\prime}$. Therefore $S^{\prime} \cong S$ as Z-PSH-algebras, with $y^{\prime} \mapsto y$ determining the unique Z-PSH-isomorphism between them. 
2. The ring $L$ and $\mathbf{P S H}$-algebras over it. As in [H-H, p. 3.8] define the $\mathbf{Z} / 2$-graded ring $L$ to be $\mathbf{Z}[\lambda] /\left(\lambda^{3}=2 \lambda\right)$ with degree $\lambda=(1) \in \mathbf{Z} / 2$. Thus

$$
L_{(1)} \cong \mathbf{Z} \text { with generator } \lambda
$$

and

$$
L_{(0)} \cong \mathbf{Z} \oplus \mathbf{Z} \quad \text { with generators } 1 \text { and } \rho:=\lambda^{2}-1 \text {. }
$$

An $L$-PSH-algebra $K$ is defined to be an $\mathbf{N}$-graded $\mathbf{Z} / 2$-graded $L$-module with extra structure. Thus, as an abelian group,

$$
K=\bigoplus_{\substack{(i) \in \mathbf{Z} / 2 \\ j \in \mathbf{N}}} K_{(i), j}
$$

is $\mathbf{Z} / 2 \times \mathbf{N}$-graded. For each $j \in \mathbf{N}, K_{(0), j} \oplus K_{(1), j}$ is a $\mathbf{Z} / 2$-graded module over $L$ (so $L_{(s)} \cdot K_{(i), j} \subset K_{(i+s), j}$ ). The extra structure is as follows:

(i) $K$ has the structure of an $\mathrm{N}$-graded Hopf algebra over $L$ with a "twist". That is, we are given maps of $\mathbf{N}$-graded modules over $L$

$$
\begin{aligned}
m: K \otimes_{L} K & \rightarrow K, \quad \Delta: K \rightarrow K \otimes_{L} K, \\
e: L & \rightarrow K, \quad e^{*}: K \rightarrow L
\end{aligned}
$$

making $K$ into a Hopf algebra, the twist being that, in defining the algebra and coalgebra structure maps for $K \otimes_{L} K$, one uses the shuffle map:

$$
a \otimes b \otimes c \otimes d \stackrel{\text { Shu }}{\rightarrow} \rho^{\varepsilon \delta+i j} a \otimes c \otimes b \otimes d
$$

where $b \in K_{\varepsilon, i}$ and $c \in K_{\delta, j}$.

(ii) We are given a set $I$ of homogeneous elements in $K$ with the following properties: (a) $x \in I$ implies $x \neq \rho x \in I$. (b) For some $X \subset I$ satisfying both $(x \in X$ implies $\rho x \notin X)$ and $(I=X \cup \rho \cdot X)$ ( $X$ is a cross section), we have that $K$ is a free $L$-module with basis $X$. It follows that any cross section is a basis over $L$, and that $K$ is a free abelian group with basis $I \cup \lambda I=X \cup \rho X \cup \lambda X$.

Elements of $I$ are referred to as irreducibles. Furthermore

$$
K \stackrel{1-\rho}{\rightarrow} K \stackrel{\lambda}{\rightarrow} K \stackrel{1-\rho}{\rightarrow} K
$$

is an exact sequence. By requiring $X$ to be an orthonormal basis, we obtain a symmetric inner product $\langle\rangle:, K \otimes_{L} K \rightarrow L$ which is independent of choice of cross section $X$ for given $I$.

Axioms connecting these structures are $(\mathrm{P})$ and $(\mathrm{S})$ below:

(P) The maps $m, \Delta, e, e^{*}$ are all positive maps in that the set of nonnegative integer linear combinations of the above $\mathbf{Z}$-basis and the obvious derived one in $K \otimes_{L} K$ are preserved.

(S) The pairs $(m, \Delta)$ and $\left(e, e^{*}\right)$ of morphisms are adjoint with respect to $\langle$,$\rangle ,$ using $\langle\langle a \otimes b, c \otimes d\rangle\rangle=\langle a, c\rangle\langle b, d\rangle$ to determine the derived inner product on $K \otimes_{L} K$. Note that $\langle$,$\rangle is strictly symmetric and that the shuffle map is used without the$ $\operatorname{sign} \rho^{\varepsilon \delta+i j}$ in defining $\langle\langle\rangle$,$\rangle .$ 
PROPOSITION 2.1. Associativity of $m$ and $\Delta$ follow from the other assumptions. Futhermore $m$ and $\Delta$ are pseudo-commutative in that $x y=\rho^{\varepsilon \delta+i j} y x$ for all $x \in K_{\varepsilon, i}$ and $y \in K_{\delta, j}$ and dually for $\Delta$ :

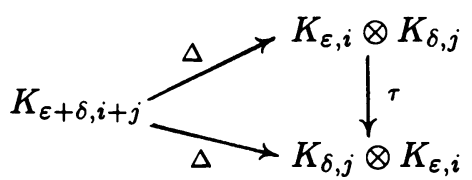

commutes, where $\tau(x \otimes y)=\rho^{\varepsilon \delta+i j} y \otimes x$ for $x \in K_{\varepsilon, i}, y \in K_{\delta, j}$. Note that $\rho$ is not -1 .

This follows exactly as in $[\mathbf{Z}, 1.6]$.

DEFINITION. An $L$-PSH-algebra $K$ is an atom iff $P \cap X$ is a singleton for a cross section $X$ in $I$, where $P$ is the set of primitives for $\Delta$. (Equivalently $P \cap I$ is a doubleton, invariant under $\rho$, so this is independent of choice of $X$.)

PROPOSITION 2.2. Any tensor product of L-PSH-algebras has again the structure of an L-PSH-algebra if we define the set of irreducibles to be

$$
\left\{x_{\alpha_{1}} \otimes x_{\alpha_{2}} \otimes \cdots \otimes x_{\alpha_{n}}: n \geq 1, x_{\alpha_{i}} \in I_{K_{\alpha_{i}}}\right\} .
$$

The proof is straightforward. Note that products and coproducts are defined using the "sign" $\rho^{\varepsilon \delta+i j}$ when shuffles take place (whereas this does not apply to the inner product).

THEOREM 2.3. Every L-PSH-algebra is isomorphic to a tensor product of atoms.

This will be proved in the next section using methods similar to Zelevinski's.

THEOREM 2.4. For each $((i), j)$ there is up to isomorphism exactly one atomic $L$-PSH-algebra $K$ whose irreducible primitive $k_{1}$ has degree $((i), j)$.

If $i+j$ is even, there exist irreducibles $k_{n} \in K_{(\text {in }), j n}$ such that

(i) $K \cong L\left[k_{1}, k_{2}, \ldots\right]$ as an L-algebra;

(ii) $\Delta\left(k_{n}\right)=\sum_{s=0}^{n} k_{s} \otimes k_{n-s}$ (where $\left.k_{0}=1\right)$.

If $i+j$ is odd, for $n>0$ there exist irreducibles

$$
k_{n} \in \begin{cases}K_{(n+1), j n} & (i \text { even }) \\ K_{(1), j n} & (i \text { odd })\end{cases}
$$

such that

(i) $K \cong \operatorname{Alg}_{L}\left\{k_{1}, k_{2}, \ldots\right\} / I$, where $I$ is the ideal generated by

$$
k_{n}^{2}-(-1)^{n+1} \lambda\left(k_{2 n}+\lambda \sum_{s=1}^{n-1}(-1)^{s} k_{s} k_{2 n-s}\right) \text { "squaring relations" }
$$

and by

$$
\left\{\begin{array}{ll}
k_{n} k_{m}-\rho^{m+n+1} k_{m} k_{n} & (i \text { even }) \\
k_{n} k_{m}-\rho k_{m} k_{n} & (i \text { odd })
\end{array}\right. \text { "pseudocommutativity relations", }
$$

(ii) $\Delta\left(k_{n}\right)=k_{n} \otimes 1+1 \otimes k_{n}+\lambda \sum_{s=1}^{n-1} k_{s} \otimes k_{n-s}$.

This will be proved in $\S 4$. 
3. Proof of Theorem 2.3. Let $(K, I, m, \Delta)$ be any $L$-PSH-algebra. Choose a cross section $X \subset I$. Let $P$ be the $L$-module of $\Delta$-primitives in $K$. For $x \in P \cap X$, define $I_{(x)}:=\left\{y \in I: \exists n \geq 0\right.$ with $\left.\left\langle y, x^{n}\right\rangle \neq 0\right\}$. Then $X_{(x)}:=I_{(x)} \cap X$ is a cross section of $I_{(x)}$. Let $K_{(x)}:=\operatorname{Span}_{L} I_{(x)}=\operatorname{Span}_{L} X_{(x)}$. Then we shall prove:

(A) $\left(K_{(x)}, I_{(x)}, m\left|K_{(x)} \otimes K_{(x)}, \Delta\right| K_{(x)}\right)$ is a sub-L-PSH-algebra of $K$.

(B) Multiplication defines an isomorphism $\bigotimes_{x \in P \cap X} K_{(x)} \rightarrow K$ of $L$-PSHalgebras.

LEMMA 3.1. Suppose $Y \subset P$ and satisfies $\left\langle y, y^{\prime}\right\rangle=0$ for all elements $y \neq y^{\prime}$ in $Y$ (for example $Y=P \cap X)$. Then if all $y_{i}, y_{i}^{\prime}$ are in $Y$, we have $\left\langle y_{1} \cdots y_{r}, y_{1}^{\prime} \cdots y_{s}^{\prime}\right\rangle$ $=0$ unless $r=s$ and $\left(y_{1}^{\prime}, \ldots, y_{s}^{\prime}\right)$ is a re-arrangement of $\left(y_{1}, \ldots, y_{r}\right)$.

ProOF. Proceed by induction on $r$. For $r=1$, the case $s=1$ is a hypothesis. If $s>1$, we get

$$
\begin{aligned}
\left\langle y_{1}, y_{1}^{\prime} \cdots y_{s}^{\prime}\right\rangle & =\left\langle\left\langle y_{1} \otimes 1+1 \otimes y_{1}, y_{1}^{\prime} \otimes\left(y_{2}^{\prime} y_{3}^{\prime} \cdots y_{s}^{\prime}\right)\right\rangle\right\rangle \\
& =\left\langle y_{1}, y_{1}^{\prime}\right\rangle\left\langle 1, y_{2}^{\prime} \cdots y_{s}^{\prime}\right\rangle+\left\langle 1, y_{1}^{\prime}\right\rangle\left\langle y_{1}, y_{2}^{\prime} \cdots y_{s}^{\prime}\right\rangle \\
& =\left\langle y_{1}, y_{1}^{\prime}\right\rangle \cdot 0+0\left\langle y_{1}, y_{2}^{\prime} \cdots y_{s}^{\prime}\right\rangle=0 .
\end{aligned}
$$

For the inductive step

$$
\begin{aligned}
\left\langle y_{1} y_{2} \cdots y_{r}, y_{1}^{\prime} y_{2}^{\prime} \cdots y_{s}^{\prime}\right\rangle & =\left\langle\left\langle\Delta\left(y_{1} \cdots y_{r}\right), y_{1}^{\prime} \otimes\left(y_{2}^{\prime} \cdots y_{s}^{\prime}\right)\right\rangle\right\rangle \\
& =\left\langle\left\langle\prod_{i=1}^{r}\left(y_{i} \otimes 1+1 \otimes y_{i}\right), y_{1}^{\prime} \otimes\left(y_{2}^{\prime} \cdots y_{s}^{\prime}\right)\right\rangle\right\rangle \\
& =\sum\left\langle y_{i_{1}} y_{i_{2}} \cdots y_{i_{k}}, y_{1}^{\prime}\right\rangle\left\langle y_{j_{1}} \cdots y_{j_{r-k}}, y_{2}^{\prime} \cdots y_{s}^{\prime}\right\rangle \rho^{\alpha}
\end{aligned}
$$

where $\rho^{\alpha}$ is an irrelevant factor depending on $\left(i_{1}, \cdots, i_{k}\right)$ and the summation is over all subsequences $\left(i_{1}, \ldots, i_{k}\right)$ of $(1, \ldots, r)$ with $\left(j_{1}, \ldots, j_{r-k}\right)$ being the complementary subsequence. By symmetry and the inductive hypothesis, $\left\langle y_{i_{1}} \cdots y_{i_{k}}, y_{1}^{\prime}\right\rangle=0$ except when $k=1$ and $y_{1}^{\prime}=y_{i_{1}}$. The summation reduces to

$$
\sum\left\langle y_{1} y_{2} \cdots y_{i_{1}-1} y_{i_{1}+1} \cdots y_{r}, y_{2}^{\prime} \cdots y_{s}^{\prime}\right\rangle \rho^{\alpha}
$$

summation over all $i_{1}$ for which $y_{i_{1}}=y_{1}^{\prime}$. The result is now clear by induction.

Choose some arbitrary linear order for $P \cap X$. For $\omega: P \cap X \rightarrow \mathrm{N}$ with finite support, define $\pi_{\omega}=\prod_{x \in P \cap X} x^{\omega(x)}$ multiplied in the order given. $\pi_{\omega}$ depends on the ordering and on choice of $X$ only up to a factor $\rho \in L$. Let

$$
X_{\omega}=\left\{x \in X:\left\langle x, \pi_{\omega}\right\rangle \neq 0\right\} .
$$

Let

$$
K_{\omega}=\operatorname{Span}_{L} X_{\omega}
$$

Then $K_{\omega}$ is independent of choice of $X$ and of ordering chosen.

LEMMA 3.2. (i) $X$ is the disjoint union of the $X_{\omega} . X_{(x)}$ is the disjoint union of $X_{\omega}$ for $\omega$ with support $\subset\{x\}$ (that is, $\omega\left(x^{\prime}\right)=0$ for $x^{\prime} \neq x$ ).

(ii) $K=\bigoplus_{\omega} K_{\omega} ; K_{(x)}=\bigoplus_{\text {support } \omega \subset\{x\}} K_{\omega}$.

(iii) $K_{\omega^{\prime}} \cdot K_{\omega^{\prime \prime}} \subset K_{\omega^{\prime}+\omega^{\prime \prime}}$.

(iv) $\Delta\left(K_{\omega}\right) \subset \bigoplus_{\left\{\left(\omega^{\prime}, \omega^{\prime \prime}\right): \omega^{\prime}+\omega^{\prime \prime}=\omega\right\}} K_{\omega^{\prime}} \otimes K_{\omega^{\prime \prime}}$. 
PROOF. (i) To prove disjointness, suppose $x_{0} \in X$ and $\left\langle x_{0}, \pi_{\omega}\right\rangle \neq 0 \neq\left\langle x_{0}, \pi_{\omega^{\prime}}\right\rangle$. By positivity

$$
\pi_{\omega}=\sum_{X} \alpha_{x} x, \quad \pi_{\omega^{\prime}}=\sum_{X} \alpha_{x}^{\prime} x
$$

for certain $\alpha_{x}$ and $\alpha_{x}^{\prime}$ in $L_{+}:=\mathbf{N} \cdot 1+\mathbf{N} \cdot \rho+\mathbf{N} \cdot \lambda \subset L$. Let $L^{\#}$ denote the set of nonzero elements in $L_{+}$. Since

$$
L^{\#}+L_{+} \subset L^{\#} \supset\left(L^{\#}\right) \cdot\left(L^{\#}\right)
$$

and by assumption $\alpha_{x_{0}}$ and $\alpha_{x_{0}}^{\prime}$ are in $L^{\#}$, we get

$$
\left\langle\pi_{\omega}, \pi_{\omega^{\prime}}\right\rangle=\alpha_{x_{0}} \alpha_{x_{0}}^{\prime}+\sum_{x \neq x_{0}} \alpha_{x} \alpha_{x}^{\prime}
$$

is in $L^{\#}+L_{+} \subset L^{\#}$. Thus $\left\langle\pi_{\omega}, \pi_{\omega^{\prime}}\right\rangle \neq 0$, so $\omega=\omega^{\prime}$ by 3.1 as required. To prove $X=\bigcup_{\omega} X_{\omega}$, suppose $x_{0} \in X \backslash\left(\bigcup_{\omega} X_{\omega}\right)$ and $x_{0}$ has smallest grading among elements with this property. Now $x_{0} \notin P$ since then $x_{0} \in X_{\omega}$ for that $\omega$ sending $x_{0}$ to 1 and all other $x$ in $X \cap P$ to 0 . Write the coproduct

$$
\Delta x_{0}=x_{0} \otimes 1+1 \otimes x_{0}+\sum \mu_{x^{\prime}, x^{\prime \prime}} x^{\prime} \otimes x^{\prime \prime}
$$

summation over certain $\left(x^{\prime}, x^{\prime \prime}\right)$ in $X$ with $\operatorname{deg} x^{\prime}<\operatorname{deg} x_{0}>\operatorname{deg} x^{\prime \prime}$. By positivity each $\mu_{x^{\prime}, x^{\prime \prime}} \in L_{+}$and for some $\left(x_{0}^{\prime}, x_{0}^{\prime \prime}\right), \mu_{x_{0}, x_{0}^{\prime \prime}} \neq 0$ since $x_{0} \notin P$. By choice of $x_{0}$, for some $\omega^{\prime}, \omega^{\prime \prime}$ we have $x_{0}^{\prime} \in X_{\omega^{\prime}}, x_{0}^{\prime \prime} \in X_{\omega^{\prime \prime}}$. But then

$$
\left\langle x_{0}^{\prime}, \pi_{\omega^{\prime}}\right\rangle \in L^{\#}, \quad\left\langle x_{0}^{\prime \prime}, \pi_{\omega^{\prime \prime}}\right\rangle \in L^{\#} .
$$

Thus for some irrelevant $\alpha$,

$$
\begin{aligned}
\left\langle x_{0}, \pi_{\omega^{\prime}+\omega^{\prime \prime}}\right\rangle & =\left\langle x_{0}, \rho^{\alpha} \pi_{\omega^{\prime}} \pi_{\omega^{\prime \prime}}\right\rangle \\
& =\rho^{\alpha}\left\langle\left\langle\Delta x_{0}, \pi_{\omega^{\prime}} \otimes \pi_{\omega^{\prime \prime}}\right\rangle\right\rangle \\
& =\rho^{\alpha} \sum \mu_{x^{\prime}, x^{\prime \prime}}\left\langle x^{\prime}, \pi_{\omega^{\prime}}\right\rangle\left\langle x^{\prime \prime}, \pi_{\omega^{\prime \prime}}\right\rangle .
\end{aligned}
$$

Each factor $\mu_{x^{\prime}, x^{\prime \prime}},\left\langle x^{\prime}, \pi_{\omega^{\prime}}\right\rangle$ and $\left\langle x^{\prime \prime}, \pi_{\omega^{\prime \prime}}\right\rangle$ is in $L_{+}$, and $\mu_{x_{0}^{\prime}, x_{0}^{\prime \prime}},\left\langle x_{0}^{\prime}, \pi_{\omega^{\prime}}\right\rangle,\left\langle x_{0}^{\prime \prime}, \pi_{\omega^{\prime \prime}}\right\rangle$ are nonzero, so $\left\langle x_{0}, \pi_{\omega^{\prime}+\omega^{\prime \prime}}\right\rangle \neq 0$. This shows $x_{0} \in X_{\omega^{\prime}+\omega^{\prime \prime}}$, a contradiction establishing that $X=\bigcup_{\omega} X_{\omega}$. The definition of $X_{(x)}$ shows that it is the union of those $X_{\omega}$ for which $\omega x^{\prime}=0$ for all $x^{\prime} \neq x$.

(ii)

$$
\begin{gathered}
K=\operatorname{Span}_{L} X=\operatorname{Span}_{L}\left(\bigcup_{\omega} X_{\omega}\right) \\
\underset{(*)}{=} \bigoplus_{\omega} \operatorname{Span}_{L}\left(X_{\omega}\right)=\bigoplus_{\omega} K_{\omega},
\end{gathered}
$$

where $(*)$ holds because of disjointness and freeness. Similarly

$$
K_{(x)}=\bigoplus_{\substack{\omega x^{\prime}=0 \\ \forall x^{\prime} \neq x}} K_{\omega} .
$$


(iii) It suffices to show $X_{\omega^{\prime}} \cdot X_{\omega^{\prime \prime}} \subset K_{\omega^{\prime}+\omega^{\prime \prime}}$. We show that if $x^{\prime} \in X_{\omega^{\prime}}, x^{\prime \prime} \in X_{\omega^{\prime \prime}}$, $x \in X$ and $\left\langle x^{\prime} x^{\prime \prime}, x\right\rangle \neq 0$, then $\left\langle x, \pi_{\omega^{\prime}+\omega^{\prime \prime}}\right\rangle \neq 0$. It suffices to show $\left\langle\left\langle\Delta x, \pi_{\omega^{\prime}} \otimes\right.\right.$ $\left.\left.\pi_{\omega^{\prime \prime}}\right\rangle\right\rangle \neq 0$, which follows since $\Delta x$ is an $L_{+}$-linear combination of $\left\{x_{1} \otimes x_{2}: x_{i} \in X\right\}$ where the coefficient of $x^{\prime} \otimes x^{\prime \prime}$ is nonzero, since it is $\left\langle\left\langle\Delta x, x^{\prime} \otimes x^{\prime \prime}\right\rangle\right\rangle=\left\langle x, x^{\prime} x^{\prime \prime}\right\rangle$.

(iv) is immediate from (iii) and self-duality.

\section{LEMMA 3.3. Multiplication gives an isomorphism}

$$
K_{\omega^{\prime}} \otimes_{L} K_{\omega^{\prime \prime}} \rightarrow K_{\omega^{\prime}+\omega^{\prime \prime}}
$$

for all $\left(\omega^{\prime}, \omega^{\prime \prime}\right)$ with disjoint support (i.e. $\omega^{\prime} x \neq 0$ implies $\omega^{\prime \prime} x=0$.)

PROOF. Elements $x^{\prime} \otimes x^{\prime \prime}$, with $x^{\prime} \in X_{\omega^{\prime}}$ and $x^{\prime \prime} \in X_{\omega^{\prime \prime}}$, of an $L$-basis for $K_{\omega^{\prime}} \otimes_{L} K_{\omega^{\prime \prime}}$ map to $\rho^{\alpha} x^{\prime} x^{\prime \prime}$ for some $\alpha$. Thus it suffices to prove that if $\omega^{\prime}$ and $\omega^{\prime \prime}$ have disjoint support, then:

(i) $X_{\omega^{\prime}+\omega^{\prime \prime}}=\left\{\rho^{\beta\left(x^{\prime}, x^{\prime \prime}\right)} x^{\prime} x^{\prime \prime}: x^{\prime} \in X_{\omega^{\prime}}, x^{\prime \prime} \in X_{\omega^{\prime \prime}}\right\}$ for some choices of $\beta\left(x^{\prime}, x^{\prime \prime}\right)$ $=0$ or 1 .

(ii) If $\left\{x^{\prime} x^{\prime \prime}, \rho x^{\prime} x^{\prime \prime}\right\}=\left\{y^{\prime} y^{\prime \prime}, \rho y^{\prime} y^{\prime \prime}\right\}$ for $x^{\prime}, y^{\prime}$ in $X_{\omega^{\prime}}$ and $x^{\prime \prime}, y^{\prime \prime}$ in $X_{\omega^{\prime \prime}}$ then $x^{\prime}=y^{\prime}$ and $x^{\prime \prime}=y^{\prime \prime}$.

But (i) and (ii) follow easily from

(iii) $\left\langle x^{\prime} x^{\prime \prime}, y^{\prime} y^{\prime \prime}\right\rangle=\left\langle x^{\prime}, y^{\prime}\right\rangle \cdot\left\langle x^{\prime \prime}, y^{\prime \prime}\right\rangle$ for $x^{\prime}$ and $y^{\prime}$ in $X_{\omega^{\prime}}, x^{\prime \prime}$ and $y^{\prime \prime}$ in $X_{\omega^{\prime \prime}}$, with $\omega^{\prime}$ and $\omega^{\prime \prime}$ of disjoint support.

Deduction of (ii) is immediate. To deduce (i), we have $x^{\prime} x^{\prime \prime}$ positive and $\left\langle x^{\prime} x^{\prime \prime}, x^{\prime} x^{\prime \prime}\right\rangle$ $=1$ so $x^{\prime} x^{\prime \prime} \in I$. Thus $x^{\prime} x^{\prime \prime}$ or $\rho x^{\prime} x^{\prime \prime}$ is in $X$, and therefore in $X_{\omega^{\prime}+\omega^{\prime \prime}}$ by 3.2. These products exhaust all of $X_{\omega^{\prime}+\omega^{\prime \prime}}$ since $\pi_{\omega^{\prime}+\omega^{\prime \prime}}=\rho^{\alpha} \pi_{\omega^{\prime}} \pi_{\omega^{\prime \prime}}$ is a linear combination of $\left\{x^{\prime} x^{\prime \prime}: x^{\prime} \in X_{\omega^{\prime}}, x^{\prime \prime} \in X_{\omega^{\prime \prime}}\right\}$. To establish (iii) write

$$
\Delta x^{\prime}=\sum_{\substack{z \in X \\ w \in X}} \alpha_{z, w}^{\prime} z \otimes w, \quad \Delta x^{\prime \prime}=\sum_{\substack{u \in X \\ v \in X}} \alpha_{u, v}^{\prime \prime} u \otimes v
$$

where all $\alpha_{z, w}^{\prime}$ and $\alpha_{u, v}^{\prime \prime}$ are in $L_{+}$. Then

$$
\begin{aligned}
\left\langle x^{\prime} x^{\prime \prime}, y^{\prime} y^{\prime \prime}\right\rangle & =\left\langle\left\langle\Delta\left(x^{\prime} x^{\prime \prime}\right), y^{\prime} \otimes y^{\prime \prime}\right\rangle\right\rangle \\
& =\sum_{u, v, w, z} \alpha_{z, w}^{\prime} \alpha_{u, v}^{\prime \prime}\left\langle z u, y^{\prime}\right\rangle\left\langle w v, y^{\prime \prime}\right\rangle .
\end{aligned}
$$

By 3.2(iv),

$$
\begin{aligned}
& \alpha_{z, w}^{\prime}=0 \text { unless } z \in X_{\omega_{1}}, w \in X_{\omega_{2}} \text { with } \omega_{1}+\omega_{2}=\omega^{\prime} \\
& \alpha_{u, v}^{\prime \prime}=0 \text { unless } u \in X_{\omega_{3}}, v \in X_{\omega_{4}} \text { with } \omega_{3}+\omega_{4}=\omega^{\prime \prime}
\end{aligned}
$$

Then

$$
z u \in K_{\omega_{1}+\omega_{3}}, \text { so }\left\langle z u, y^{\prime}\right\rangle=0 \text { unless } \omega_{1}+\omega_{3}=\omega^{\prime}
$$

and

$$
w v \in K_{\omega_{2}+\omega_{4}} \text {, so }\left\langle w v, y^{\prime \prime}\right\rangle=0 \text { unless } \omega_{2}+\omega_{4}=\omega^{\prime \prime} .
$$

Thus $\left\langle x^{\prime} x^{\prime \prime}, y^{\prime} y^{\prime \prime}\right\rangle=0$ unless all four equations hold. But then $\omega_{2}=\omega_{3}$. By disjointness of supports of $\omega^{\prime}$ and $\omega^{\prime \prime}$, we find $\omega_{2}=\omega_{3}$ is zero. So $w=u=1$, $z=x^{\prime}, v=x^{\prime \prime}, \alpha_{z, w}^{\prime}=1, \alpha_{u, v}^{\prime \prime}=1$. This completes verification of (iii) and proof of 3.3 .

To complete the proof of 2.3, first consider (A). That $K_{(x)}$ is a sub-Hopf-algebra of $K$ is immediate from 3.2(ii), (iii) and (iv). By 3.2(i), $X_{(x)}$ is a basis for $K_{(x)}$ over 
$L$ such that the associated inner product is $\left.\langle\rangle\right|_{,K_{(x)} \otimes K_{(x)}}$. Axioms (P) and (S) are immediate for $K_{(x)}$. Finally to check (B), injectivity of the map is immediate from 3.3 since every element of $\bigotimes_{x \in P \cap X} K_{(x)}$ (defined by direct limits if $P \cap X$ is infinite) is in the image of $K_{\left(x_{1}\right)} \otimes K_{\left(x_{2}\right)} \otimes \cdots \otimes K_{\left(x_{r}\right)}$ for some sequence of distince $x_{i} \in P \cap X$. Surjectivity also follows from 3.3, since $K=\bigoplus K_{\omega}$ and $K_{\omega}$ is in the image under multiplication of $K_{\left(x_{1}\right)} \otimes K_{\left(x_{2}\right)} \otimes \cdots \otimes K_{\left(x_{r}\right)}$ where support $\omega=\left\{x_{1}, \ldots, x_{r}\right\}$.

This completes the proof of Theorem 2.3. Referring back to (A) and (B) before 2.3 and to the definition of $K_{(x)}$, we have the following important corollary to the proof.

COROLLARY 3.4. If $K$ is atomic with irreducible primitive $x$, and $y$ is any irreducible, then for some integer $n \geq 0$ we have $\left\langle y, x^{n}\right\rangle \neq 0$.

\section{Structure of atoms-Proof of $\mathbf{2 . 4}$.}

LEMMA 4.1. The four cases $((i), j)=((0), 1),((0), 2),((1), 1)$, and $((1), 2)$ imply all the other cases in Theorem 2.4 .

PrOOF. An atom $K$ with irreducible primitive in degree $((i), j)$ will be trivial in all dimensions $\left(\left(i^{\prime}\right), j^{\prime}\right)$ for which $j \nmid j^{\prime}$ by Corollary 3.4. If $j$ is odd we may divide all Z-degrees by $j$, and if $j$ is even, by $j / 2$. The result is still an $L$-PSH-algebra since the pseudocommutativity is sensitive only to $j(\bmod 2)$. Thus we have a 1-1 correspondence between atoms with parameters $((i), j)$ and those in one of the four cases, as required.

LEMMA 4.2. There exist four L-PSH-algebras as described in Theorem 2.4 with gradings as in 4.1 .

PROOF. In [H-H2], double coverings of $H>S_{n}$ are constructed. Suitable sequences of these (varying $n$ ) give $L$-PSH algebras when the functor $T^{*}$ of that paper is applied. The data for such a construction includes coverings $G$ for $H$ and $S_{n}^{\prime}$ for $S_{n}$. As noted in [H-H2] we obtain a unique irreducible primitive for each of the four choices below, these primitives having the correct grading and the algebras having the correct structure. When $j=1$, take $S_{n}^{\prime}$ to be the essential covering $\tilde{S}_{n}$ studied in [H-H1] with the usual sign homomorphism. When $j=2$, take $S_{n}^{\prime}$ to be $S_{n} \times C_{2}$ with left projection and use the zero map for the "sign". When $i=0$, take $H$ to be the trivial group, so $G$ is its unique double cover $C_{2}$ with necessarily zero "sign". When $i=1$, take $H$ to be $C_{2}$ and $G$ to be $C_{4}$, with nonzero sign. In each case we find that $T^{*} G$ is free over $L$ on one irreducible in the appropriate grading, giving the required irreducible primitive.

NOTE. It is only the case $(i, j)=(1,2)$ which requires the construction of the covers in $[\mathbf{H}-\mathbf{H 2}]$. When $(i, j)=(0,1)$, the original sequence $\tilde{S}_{n}$ of covers constructed by Schur gives the atomic PSH-algebra by the work in [H-H1]. The other two cases can be verified simply by tensoring $L$ with the Z-PSH-atom studied by Zelevinski. Note that Zelevinski also does not directly construct his atom, but (presumably) relies on the ring $\bigoplus_{n} R\left(S_{n}\right)$ to prove existence. Direct combinatorial construction in all cases seems rather difficult. Our proof of the classification of atoms, namely 2.4, definitely depends on the existence of atoms. A similar proof can be used to replace Zelevinski's proof with a superficially shorter one, first using, 
for example, Liulevicius' Hopf algebra method [L] to establish existence of the atom (i.e. to establish that $\bigoplus_{n} R\left(S_{n}\right)$ is an atomic PSH-algebra with the correct structure). Zelevinski's more combinatorial argument (to prove uniqueness of atomic PSH-algebras up to regrading) uses an argument with determinants reminiscent of a classical argument in the representation theory of the symmetric group. It would be very interesting to see a similar more elementary proof of 2.4 not depending on the existence of atoms with the stated structure.

In 4.3, 4.4 and the proof of 2.4 below, we shall denote the particular atoms constructed above in 4.2 as $H$ with generators $h_{i}$. A general atom, which we wish to prove isomorphic to one such $H$, will be denoted $K$ with generators $k_{i}$.

DEFINITION. We say an element $x$ of a PSH-algebra $K$ is $\rho$-unique with respect to a property if the only other element with the property is $\rho x$ (including instances where $\rho x=x$ ).

LEMMA 4.3. Let $K$ be an atom whose irreducible primitive $k_{1}$ is in degree $((i), j)$. Then there is a sequence of irreducible elements $\left(k_{1}, k_{2}, \ldots\right)$ with the following property:

$$
k_{1} k_{n-1}= \begin{cases}\lambda k_{n}+u_{n} & \text { if } i+j \text { is odd }, \\ k_{n}+u_{n} & \text { if } i+j \text { is even, }\end{cases}
$$

where $u_{2}=0$ when $i+j$ is odd, and $u_{n}$ is irreducible and $u_{n} \neq k_{n}$ in all other caes. In the case $i+j$ odd, the $k_{n}$ are $\rho$-unique with respect to irreducibility and satisfying $(*)$. Furthermore

(i)

$$
\Delta k_{n}= \begin{cases}k_{n} \otimes 1+1 \otimes k_{n}+\lambda \sum_{s=1}^{n-1} k_{s} \otimes k_{n-s} & (i+j \text { odd }), \\ \sum_{s=0}^{n} k_{s} \otimes k_{n-s}, \text { where } k_{0}=1 & (i+j \text { even }) .\end{cases}
$$

(ii) If $i+j$ is odd, we have

$$
k_{n}^{2}=(-1)^{n+1} \lambda\left[k_{2 n}+\lambda \sum_{s=1}^{n-1}(-1)^{s-1} k_{s} k_{2 n-s}\right] \text {. }
$$

PrOof. The proof in the case where $i+j$ is even is very similar to Zelevinski's [Z, pp. 29-31] with minor modifications to work over $L$ rather than $\mathbf{Z}$. We shall leave this to the reader and concentrate on the case $i+j$ odd which is harder but does follow the pattern of Zelevinski's proof. It is convenient to introduce the notation

$$
x^{*}: K \stackrel{\Delta}{\rightarrow} K \otimes_{L} K \stackrel{1 \otimes\langle, x\rangle}{\longrightarrow} K \otimes_{L} L \cong K .
$$

Now

$$
\begin{aligned}
\left\langle k_{1}^{2}, k_{1}^{2}\right\rangle & =\left\langle\left\langle k_{1} \otimes k_{1}, \Delta k_{1}^{2}\right\rangle\right\rangle \\
& =\left\langle\left\langle k_{1} \otimes k_{1},\left(k_{1} \otimes 1+1 \otimes k_{1}\right)^{2}\right\rangle\right\rangle \\
& =\left\langle\left\langle k_{1} \otimes k_{1},(1+\rho) k_{1} \otimes k_{1}\right\rangle\right\rangle=1+\rho .
\end{aligned}
$$

(We get $1+\rho$ rather than 2 because $i+j$ is odd.) Expressing the positive element $k_{1}^{2}$ in terms of irreducibles, we must have $k_{1}^{2}=\lambda k_{2}$ for some irreducible $k_{2}$, since the only "sum of squares of positives" in $L$ giving $1+\rho$ is $\lambda^{2}=1+p$. (Note. There are several later arguments similar to this in this proof. In each case one simply looks at the coefficients of $1, \rho$ and $\lambda$ to reduce it to questions of expressing 
integers as sums of squares in $\mathbf{Z}$, each of which can be answered by (sometimes tedious) case by case consideration. Details will be suppressed.) We must have $\Delta k_{2}=k_{2} \otimes 1+a \lambda k_{1} \otimes k_{1}+1 \otimes k_{2}$ for some nonnegative $a \in \mathbf{Z}$. Using $\left\langle k_{1}^{2}, k_{2}\right\rangle=\lambda$ we see that $a=1$. As above, a simple calculation yields $\left\langle k_{1} k_{2}, k_{1} k_{2}\right\rangle=\rho+2$. Since the only sum of squares of positives yielding $\rho+2$ is $\lambda^{2}+1^{2}=\rho+2$, we find $k_{1} k_{2}=\lambda k_{3}+u_{3}$ for a pair of distinct $\rho$-unique irreducibles $k_{3}$ and $u_{3}$. It is convenient for the induction below to proceed, as above, to find distinct irreducibles $k_{4}, u_{4}$ with $k_{1} k_{3}=\lambda k_{4}+u_{4}$. For $n=2,3,4$ we find

$$
\Delta k_{n}=1 \otimes k_{n}+\lambda k_{1} \otimes k_{n-1}+\cdots ; \text { i.e. } k_{1}^{*} k_{n}=\lambda k_{n-1} .
$$

Now proceed inductively. Suppose $n \geq 5$ and for $2<m<n$ we have found irreducibles $k_{m}, u_{m}$ for which $k_{1} k_{m-1}=\lambda k_{m}+u_{m}$ and $k_{1}^{*} k_{m}=\lambda k_{m-1}$. Then

$$
\Delta\left(k_{1} k_{n-1}\right)=\Delta\left(k_{1}\right) \Delta\left(k_{n-1}\right)
$$

yields

$$
k_{1}^{*}\left(k_{1} k_{n-1}\right)=k_{n-1}+\lambda k_{1} k_{n-2} .
$$

Thus

$$
\begin{aligned}
\left\langle k_{1} k_{n-1}, k_{1} k_{n-1}\right\rangle & =\left\langle\left\langle k_{1} \otimes k_{n-1}, k_{1} \otimes\left(k_{n-1}+\lambda k_{1} k_{n-2}\right)\right\rangle\right\rangle \\
& =\left\langle k_{1}, k_{1}\right\rangle\left\langle k_{n-1}, k_{n-1}\right\rangle+\lambda\left\langle k_{1}, k_{1}\right\rangle\left\langle k_{n-1}, k_{1} k_{n-2}\right\rangle \\
& =1+\lambda^{2}=2+\rho .
\end{aligned}
$$

As above, we find that there are $\rho$-unique irreducibles $k_{n}, u_{n}$ for which $k_{1} k_{n-1}=$ $\lambda k_{n}+u_{n}$. Now

$$
\begin{aligned}
\lambda k_{1}^{*}\left(k_{n}\right)+k_{1}^{*}\left(u_{n}\right) & =k_{1}^{*}\left(\lambda k_{n}+u_{n}\right)=k_{1}^{*}\left(k_{1} k_{n-1}\right) \\
& =k_{n-1}+\lambda\left(u_{n-1}+\lambda k_{n-1}\right)=\lambda u_{n-1}+(\rho+2) k_{n-1} .
\end{aligned}
$$

Thus

$$
k_{1}^{*}\left(u_{n}\right)=\alpha u_{n-1}+\beta k_{n-1}, \quad k_{1}^{*}\left(k_{n}\right)=\gamma u_{n-1}+\delta k_{n-1}
$$

for nonnegative $\alpha, \beta, \gamma, \delta$ in $L$ for which

$$
\begin{gathered}
\alpha+\lambda \gamma=\lambda, \\
\beta+\lambda \delta=\rho+2 .
\end{gathered}
$$

But $\lambda=\left\langle k_{1} k_{n-1}, k_{n}\right\rangle=\left\langle\left\langle k_{1} \otimes k_{n-1}, k_{1} \otimes k_{1}^{*}\left(k_{n}\right)\right\rangle\right\rangle=\delta$, so $\beta=1$ by (2). The only nonnegative solutions to (1) are easily seen to be $(\alpha, \gamma)=(0,1),(0, \rho)$ and $(\lambda, 0)$. It remains to show that the first two possibilities lead to a contradiction. Since $n \geq 5$, we calculate

$$
\left\langle k_{1}^{2} k_{n-2}, k_{1}^{2} k_{n-2}\right\rangle=7 \rho+7=7 \lambda^{2}, \quad\left\langle k_{1}^{2} k_{n-2}, k_{1} k_{n-1}\right\rangle=4 \lambda .
$$

Writing $k_{1}^{2} k_{n-2}=\phi u_{n}+\psi k_{n}+\sum_{\alpha} \theta_{\alpha} u_{\alpha}$, summation over irreducibles $u_{\alpha}$ distinct from $u_{n}$ and $k_{n}$ and with $\phi, \psi, \theta_{\alpha}$ all nonnegative, we find

$$
\phi+\psi \lambda=4 \lambda, \quad \phi^{2}+\psi^{2}+\sum_{\alpha} \theta_{\alpha}^{2}=7 \rho+7 .
$$

Considering the five possibilities $\phi=t \lambda, 0 \leq t \leq 4$, leads to a unique solution $\phi=2 \lambda, \psi=\rho+1, \theta_{\alpha}=0$ for all but one $\alpha$ and $\theta_{\alpha}=\lambda$ for that $\alpha$. This yields $k_{1}^{2} k_{n-2}=2 \lambda u_{n}+(\rho+1) k_{n}+\lambda w$ for some irreducible $w$ distinct from $u_{n}$ and $k_{n}$. 
This gives $\left\langle k_{1}^{2} k_{n-2}, k_{n}\right\rangle=\rho+1$. But the first two possibilities $(\alpha, \gamma)=\left(0, \rho^{i}\right) i=0$ or 1 would yield $k_{1}^{*}\left(k_{n}\right)=\rho^{i} u_{n-1}+\lambda k_{n-1}=\rho^{i} k_{1} k_{n-2}$, giving

$$
\begin{aligned}
\left\langle k_{1}^{2} k_{n-2}, k_{n}\right\rangle & =\left\langle\left\langle k_{1} \otimes k_{1} k_{n-2}, k_{1} \otimes \rho^{i} k_{1} k_{n-2}\right\rangle\right\rangle \\
& =\rho^{i}\left\langle k_{1} k_{n-2}, k_{1} k_{n-2}\right\rangle \\
& = \begin{cases}\rho+2 & \text { if } i=0, \\
1+2 \rho & \text { if } i=1 .\end{cases}
\end{aligned}
$$

Neither of these is $\rho+1$. Hence $(\alpha, \gamma)=(\lambda, 0)$ and in particular $k_{1}^{*} k_{n}=\lambda k_{n-1}$, completing the induction.

To prove (i), we must show for $n>1$ and $0<s<n$ that $k_{s}^{*} k_{n}=\lambda k_{n-s}$ and that $w^{*} k_{n}=0$ for all irreducibles $w$ of Z-degree $s$ other than the $k_{s}$. Now by induction on $s$, we get $\left\langle k_{1}^{s}, k_{s}\right\rangle=\lambda^{s-1}$, so $k_{1}^{s}=\lambda^{s-1} k_{s}+\sum_{\alpha} \theta_{\alpha} x_{\alpha}$ summation over all irreducibles $x_{\alpha}$ other than $k_{s}$ of Z-degree $s$. By Corollary 3.4, each $\theta_{\alpha}$ is a strictly positive element of $L$. We find that

$$
\lambda^{s} k_{n-s}=\left(k_{1}^{*}\right)^{s}\left(k_{n}\right)=\left(k_{1}^{s}\right)^{*}\left(k_{n}\right)=\lambda^{s-1} k_{s}^{*}\left(k_{n}\right)+\sum_{\alpha} \theta_{\alpha} x_{\alpha}^{*}\left(k_{n}\right)
$$

Since all terms on the right are positive, we must have

$$
k_{s}^{*}\left(k_{n}\right)=\gamma k_{n-s}, \quad x_{\alpha}^{*}\left(k_{n}\right)=\beta_{\alpha} k_{n-s}
$$

for nonnegative $\gamma$ and $\beta_{\alpha}$ in $L$ for which

$$
\lambda^{s-1} \gamma+\sum_{\alpha} \theta_{\alpha} \beta_{\alpha}=\lambda^{s} .
$$

But $\gamma=a \lambda$ for some integer $a \geq 0$. In fact $a>0$, since $k_{s}^{*}\left(k_{n}\right) \neq 0$, since

$$
\left(k_{1}^{n-s}\right)^{*}\left(k_{s}^{*}\right)\left(k_{n}\right)=k_{s}^{*}\left(k_{1}^{*}\right)^{n-s}\left(k_{n}\right)=k_{s}^{*}\left(\lambda^{n-s} k_{s}\right)=\lambda^{n-s} \neq 0 .
$$

But $a \lambda^{s}+\sum_{\alpha} \theta_{\alpha} \beta_{\alpha}=\lambda^{s}$ for positive $a \in \mathbf{Z}$, positive $\theta_{\alpha} \in \mathbf{L}$ and nonnegative $\beta_{\alpha} \in \mathrm{L}$ is only possible for $a=1$ and $\beta_{\alpha}=0$ for all $\alpha$. This completes the proof of (i).

To prove (ii), we need the following calculations which are straightforward now that $\Delta k_{n}$ is known:

$$
\begin{gathered}
\left\langle k_{s} k_{n-s}, k_{n}\right\rangle=\lambda \quad \text { for } 1 \leq s<n ; \\
\left\langle k_{s} k_{n-s}, k_{s} k_{n-s}\right\rangle=(2 s-1) \lambda^{2}+1 \quad \text { for } 1 \leq s<n / 2 ; \\
\left\langle k_{s} k_{n-s}, k_{t} k_{n-t}\right\rangle=2 s \lambda^{2} \quad \text { for } 1 \leq s<t<n / 2 ; \\
\left\langle k_{n}^{2}, k_{s} k_{2 n-s}\right\rangle=2 s \lambda^{s} \quad \text { for } 1 \leq s<n / 2 ; \\
\left\langle k_{n}^{2}, k_{n}^{2}\right\rangle=(2 n-1) \lambda^{2} ; \quad\left\langle k_{n}^{2}, k_{2 n}\right\rangle=\lambda .
\end{gathered}
$$

The required identity is immediate from the equations in the propositions below, which we state separately to provide some information on explicit decompositions into irreducibles of the basis $\left\{k_{\alpha}: \alpha \in \mathbf{D}\right\}$ in the easiest case, that is, for partitions $\alpha$ of length 2 .

Proposition 4.4. For each $n>2$, there is a finite sequence $k_{n}, y_{1}, y_{2}, \ldots$ of distinct irreducibles such that if $1 \leq s<n / 2$

$$
k_{s} k_{n-s}=y_{s}+(\rho+1) \sum_{t=1}^{s-1} y_{t}+\lambda k_{n}
$$


This equation defines the $y_{i}$ " $\rho$-uniquely." Furthermore, when $n=2 l$,

$$
k_{l}^{2}=(\rho+1) \sum_{t=1}^{l-1} y_{t}+\lambda k_{2 l} .
$$

ProOF. For $s=1$, we take $y_{1}=u_{n}$. Proceeding by induction on $s$ suppose $y_{1}, \ldots, y_{s-1}$ have been constructed, are irreducible, distinct from each other and from $k_{n}$, and such that

$$
k_{r} k_{n-r}=y_{r}+(\rho+1) \sum_{i=1}^{r-1} y_{t}+\lambda k_{n} \text { for } 1 \leq r<s .
$$

Now $\left\langle k_{s} k_{n-s}, k_{n}\right\rangle=\lambda$ and repeated application of (3) yields

$$
\left\langle k_{s} k_{n-s}, y_{r}\right\rangle=\lambda^{2}=\rho+1 \text { for } 1 \leq r<s .
$$

Thus $k_{s} k_{n-s}=w+(\rho+1) \sum_{t=1}^{s-1} y_{t}+\lambda k_{n}$ for some nonnegative $w$ for which $\left\langle w, y_{t}\right\rangle=$ 0 for $t<s$ and $\left\langle w, k_{n}\right\rangle=0$. Now

$$
(2 s-1)(\rho+1)+1=\left\langle k_{s} k_{n-s}, k_{s} k_{n-s}\right\rangle=\langle w, w\rangle+(s-1)(\rho+1)^{2}+\lambda^{2} .
$$

Thus $\langle w, w\rangle=1$, so let $y_{s}=w$. To prove the formula for $k_{l}^{2}$, a repeated application of (4) yields $\left\langle k_{l}^{2}, y_{t}\right\rangle=\rho+1$ for $1 \leq t<l$, so $k_{l}^{2}=v+(\rho+1) \sum_{t=1}^{l-1} y_{t}+\lambda k_{2 l}$ for some nonnegative $v$. But now

$$
\langle v, v\rangle+(l-1)(\rho+1)^{2}+\lambda^{2}=\left\langle k_{l}^{2}, k_{l}^{2}\right\rangle=(2 l-1) \lambda^{2},
$$

so $\langle v, v\rangle=0$ and thus $v=0$ as required.

PROOF OF THEOREM 2.4. By mapping $h_{n}$ to $k_{n}$, where $h_{n} \in H$ is an atomic PSH-algebra with the desired structure and $k_{n} \in K$, a given atom, we get in every case a unique homomorphism of graded algebras $\theta: H \rightarrow K$. (In the case of $i+j$ odd, the $k_{n}$ satisfy the pseudocommutativity relations by definition and the squaring relations by 3.3 , and the $h_{n}$ satisfy no other independent relation, so $\theta$ is well defined). We wish to prove that $\theta$ is an isomorphism of $L$-PSH-algebras. Because the formulae for $\Delta h_{n}$ and $\Delta k_{n}$ are "the same" and $\Delta$ is an algebra homomorphism, we see that $\theta$ is a morphism of coalgebras. Furthermore, for any partitions $\alpha$ and $\beta$, with distinct parts, we have

$$
\left\langle k_{\alpha}, k_{\beta}\right\rangle_{K}=\left\langle h_{\alpha}, h_{\beta}\right\rangle_{H},
$$

basically because these are calculated using the formula for $\Delta$. (For example, when $i+j$ is odd, the answer is $\sum_{A} \lambda^{n(A)}$, summation over the set of matrices with nonnegative integer entries, row sums $\alpha$, column sums $\beta$, with $n(A)$ equal to twice the number of positive entries in $A$ less the length of $\alpha$ less the length of $\beta$.) Thus we have $\langle\theta a, \theta b\rangle_{K}=\langle a, b\rangle_{H}$ for all $a$ and $b$ in $H$. Now let

$$
\left\{s_{\alpha} \cdot\left\{\begin{array}{l}
\alpha \in \mathbf{P} \text { when } i+j \text { is even } \\
\alpha \in \mathbf{D} \text { when } i+j \text { is odd }
\end{array}\right\}\right\}
$$

be the $\rho$-unique orthonormal basis over $L$ for $H$. Here $\mathbf{P}$ is the set of all partitions, $\mathbf{D}$ of all partitions into distinct parts. Thus $\left\{\theta s_{\alpha}\right\}$ is an orthonormal basis for $\operatorname{Im} \theta$. In particular, $\theta$ is a monomorphism and $\operatorname{Im} \theta$ is a $T$-submodule of $K$. But by Corollary 3.4, any irreducible $x \in K$ satisfies $\left\langle x, k_{1}^{n}\right\rangle \neq 0$ for some $n$, so $x$ lies in 
$\operatorname{Im} \theta=\operatorname{Alg}\left\{k_{n}\right\}$ rather than in the $T$-complement of $\operatorname{Im} \theta$. So that complement is 0 , and $\operatorname{Im} \theta=K$. We now have that $\theta$ is an inner product preserving isomorphism of Hopf algebras. But since $\theta$ maps the positive basis $\left\{h_{\alpha}\right\}$ to the positive basis $\left\{k_{\alpha}\right\}$, it must send irreducibles to irreducibles rather than their negatives, and so $\theta$ is an isomorphism of $L$-PSH-algebras.

\section{REFERENCES}

[H] P. Hoffman, $\tau$-rings and wreath product representations, Lecture Notes in Math., vol. 746, Springer, 1979.

[H-H1] Peter Hoffman and John Humphreys, Hopf algebras and projective representations of $G$ ᄂ $S_{n}$, and $G$ ? $A_{n}$, Canad. J. Math. 38 (1986), 1380-1458.

[H-H2] _ _ Projective representations of generalized symmetric groups using PSH-algebras (to appear).

[L] A. Liulevicius, Arrows, symmetries and representation rings, J. Pure Appl. Algebra 19 (1980), 259-273.

[Z] Andrey V. Zelevinski, Representations of finite classical groups, Lecture Notes in Math., vol. 869, Springer, 1981.

Department of Pure Mathematics, University of Waterloo, Waterloo, ONTARIO, CANADA N2L 3G1 